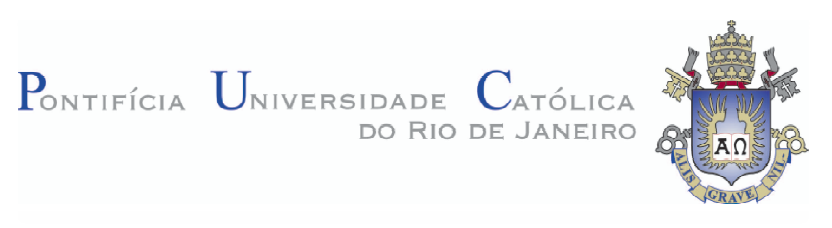

Paula de Mello Ribeiro Pinto

\title{
Desenvolvimento de Metodologia de Manipulação de Mapas de Características dos Compressores Axiais
}

\section{Dissertação de Mestrado}

Dissertação apresentada como requisito parcial para obtenção do título de Mestre pelo Programa de PósGraduação em Engenharia Mecânica da PUC-Rio.

Orientador: Prof. Sérgio Leal Braga Co-orientador: Dr. Sandro Barros Ferreira 


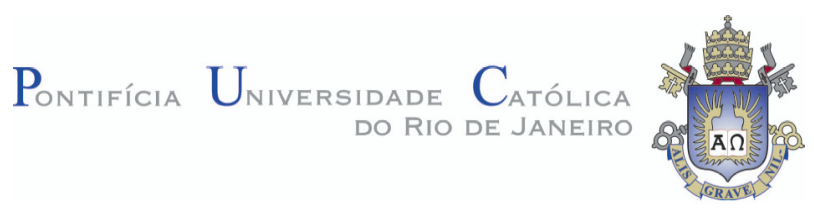

Paula de Mello Ribeiro Pinto

\section{Desenvolvimento de Metodologia de Manipulação de Mapas de Características dos Compressores Axiais}

Dissertação apresentada como requisito parcial para obtenção do título de Mestre pelo Programa de PósGraduação em Engenharia Mecânica da PUC-Rio. Aprovada pela Comissão Examinadora abaixo assinada.

\section{Prof. Sergio Leal Braga} Orientador Pontifícia Universidade Católica do Rio de Janeiro - PUC-Rio

\section{Dr. Sandro Barros Ferreira \\ Co-orientador GT2 Energia}

Prof. José Alberto dos Reis Parise Pontifícia Universidade Católica do Rio de Janeiro - PUC-Rio

Prof. João Roberto Barbosa Instituto Tecnológico de Aeronáutica - ITA

Prof. José Eugênio Leal Coordenador(a) Setorial do Centro Técnico Científico - PUC-Rio 
Todos os direitos reservados. É proibida a reprodução total ou parcial do trabalho sem autorização da universidade, da autora e do orientador.

\section{Paula de Mello Ribeiro Pinto}

Graduou-se em Engenharia Elétrica pela Pontifícia Universidade Católica do Rio de Janeiro - PUC-Rio, em 2006.

\section{Ficha Catalográfica}

Pinto, Paula de Mello Ribeiro
Desenvolvimento de metodologia de manipulação
de mapas de características dos compressores axiais /
Paula de Mello Ribeiro Pinto ; orientador: Sérgio Leal
Braga ; co-orientador: Sandro Barros Ferreira. - 2010.
76 f. : il ; 30 cm
Dissertação (Mestrado)-Pontifícia Universidade
Católica do Rio de Janeiro, Departamento de Engenharia
Mecânica, Rio de Janeiro, 2010.
Inclui bibliografia
1. Engenharia mecânica - Teses. 2. Mapas de
características. 3. Compressores axiais. 4. Simulação off-
design. I. Braga, Sérgio Leal. II. Ferreira, Sandro Barros.
III. Pontifícia Universidade Católica do Rio de Janeiro.
Departamento de Engenharia Mecânica. III. Título.

CDD: 621 
Dedico este trabalho aos meus pais, Paulo Roberto e Rosely, e aos meus irmãos, Leonardo, Gustavo e Julia. 


\section{Agradecimentos}

Ao Sandro Barros Ferreira, pelo apoio, motivação e orientação durante todo o mestrado.

Ao professor Sergio Leal Braga pela oportunidade de cursar a pós-graduação no departamento de engenharia mecânica da PUC-Rio.

A PUC-Rio pelo auxílio concedido, permitindo a realização deste trabalho.

Ao professor João Roberto Barbosa pela relevante contribuição na versão final deste trabalho.

A minha família, em especial a minha mãe Rosely e ao meu pai Paulo Roberto, que são a base de tudo o que sou e conquistei até hoje.

Ao Diogo, pelo carinho e compreensão em todas as horas.

Ao Rafael pela ajuda oferecida durante todo o trabalho.

A Carol, Cláudia, Marília, e Vinicius pela amizade e apoio.

A todos os meus amigos que sempre me apoiaram e torceram por mim.

Agradeço a Deus por estar sempre presente na minha vida. 


\section{Resumo}

Pinto, Paula de Mello Ribeiro; Braga, Sergio Leal. Desenvolvimento de Metodologia de Manipulação de Mapas de Características dos Compressores Axiais. Rio de Janeiro, 16 de Abril de 2010. 76p. Dissertação de Mestrado - Departamento de Engenharia Mecânica, Pontifícia Universidade Católica do Rio de Janeiro.

Os mapas de características do compressor axial representam o desempenho deste por toda sua faixa de operação. Estes mapas podem ser utilizados para determinar o ponto de operação na simulação off-design. $\mathrm{O}$ presente trabalho propõe uma metodologia de manipulação de mapas de características de compressores axiais, baseada nas metodologias já existentes. A rotina desenvolvida, denominada PCOMP, é capaz de obter uma nova linha de rotação, entre duas linhas adjacentes conhecidas. A distância entre duas linhas adjacentes conhecidas e a nova linha irá determinar a influência que cada uma terá nas características desta nova linha. Uma vez determinada a linha de rotação, os parâmetros são interpolados a fim de determinar a vazão mássica corrigida e eficiência isentrópica para a razão de pressão correspondente, dada na entrada da rotina. $\mathrm{O}$ método de ponderação para a determinação de novas linhas de rotação apresentou desvios menores que 3\% para a vazão mássica corrigida e eficiência isentrópica. Comparando as saídas da rotina com dados de operação de uma usina real, foram encontrados desvios menores que $1 \%$ para a eficiência isentrópica. A rotina desenvolvida foi implementada no módulo de simulação do compressor da ferramenta de simulação de turbinas a gás denominada NGGT (Natural Gas \& Gas Turbine), apresentando resultados satisfatórios.

\section{Palavras-chave}

Compressores Axiais; Mapas de Características; Ponderação; Simulação offdesign. 


\section{Abstract}

Pinto, Paula de Mello Ribeiro; Braga, Sergio Leal (Advisor). A Method for the Numerical Handling of Compressor Maps. Rio de Janeiro, 2010. 76p. MSc. Thesis - Departamento de Engenharia Mecânica, Pontifícia Universidade Católica do Rio de Janeiro.

Axial compressor maps are able to represent the performance of all its operating range. These maps are used for determining the operating point in the off-design simulation. This thesis proposes a methodology for handling maps of axial compressors, based on existing methodologies. The model, called PCOMP, is able to obtain a new rotational speed line between two adjacent lines known. The distance between the known and the new line will determine the influence that each one will have in the characteristics of the new line. Once determined the speed line, one can find the operating point interpolating the known parameters to determine the corrected mass flow and isentropic efficiency for the corresponding pressure ratio given in the entry data of the routine. The weighting method using to determinate the new rotation speed line presented deviations smaller than $3 \%$ for the corrected mass flow and isentropic efficiency. By comparing the outputs of the developed code with the operating data of a real power plant, were found deviations smaller than $1 \%$ for the isentropic efficiency. The routine was successfully implemented in a gas turbine performance computer program, called NGGT (Natural Gas \& Gas Turbine) model, which presented accurate and efficient simulations.

\section{Keywords}

Axial Compressor; Component Maps; Off-design Simulation 


\section{Sumário}

1 Introdução 15

2 Revisão Bibliográfica $\quad 17$

$\begin{array}{ll}2.1 \text { Considerações Iniciais } & 17\end{array}$

2.2 Manipulação de Mapas de Características de Compressores Axiais 18

3 Conceitos Fundamentais $\quad 25$

3.1 Máquinas de Fluxo 25

3.1.1 Turbomáquinas 26

3.1.2 Turbomáquinas Térmicas $\quad 27$

$\begin{array}{lll}3.1 .3 & \text { Turbocompressor } & 28\end{array}$

$\begin{array}{ll}3.2 \text { Compressor Axial } & 29\end{array}$

3.2.1 Análise Termodinâmica do Estágio de Compressão 31

4 Simulação Fora do Ponto de Projeto (Off-Design) 40

4.1 Análise Dimensional Aplicada a Compressores 40

4.2 Mapa de Características do Compressor Axial 41

4.2.1 Mapas de Características do Compressor Axial de Geometria Variável 44

5 Metodologia 47

5.1 Método de Ponderação da Linha de Rotação 48

5.2 Método das VIGVs 54

5.3 Código Computacional 55

6 Validação e Resultados $\quad 59$

6.1 Validação do Método de Ponderação do Modelo PCOMP 59

6.2 Resultados do Modelo PCOMP 66

7 Conclusões $\quad 71$

$\begin{array}{lll}7.1 & \text { Sugestões para Trabalhos Futuros } & 73\end{array}$

8 Referências Bibliográficas $\quad 74$ 


\section{Lista de figuras}

Figura 1.1 - Turbina a gás de um eixo, [2].

Figura 2.1 - Linhas Beta utilizadas na ferramenta computacional

desenvolvida por Kurzke [8].

Figura 2.2 - Parâmetros tabelados em função das linhas Beta

e Rotação Corrigida, [10] (modificado).

Figura 2.3 -Linhas Beta definidas por Geoff Jones e Curnock [12]

utilizando a metodologia apresentada por Kurzke [8].

Figura 2.4 - Novas linhas de rotação constante determinadas

a partir da metodologia desenvolvida por Carvalho [13]. 23

Figura 3.1 - Classificação de Turbomáquinas, [16]. 26

Figura 3.2 - llustração da diferença entre compressor de fluxo

(a) axial e (b) centrífugo,[17].

Figura 3.3 - Diagrama de velocidades nos estágios de um compressor axial, [2].

Figura 3.4 - Estágio de Compressão, [23]. 31

Figura 3.5 - Variação do diâmetro do compressor, [20]. 32

Figura 3.6 - Variação da velocidade e pressão no compressor, [20]. 32

Figura 3.7 - Variação da temperatura no estágio de compressão, [21]. 34

Figura 3.8 - Triângulo de Velocidades, [2]. 37

Figura 4.1 - Mapa de características do compressor axial:

(a) razão de pressão versus vazão mássica corrigida,

(b) eficiência isentrópica versus vazão mássica, [13].

Figura 4.2 - Mapa de Características do Compressor Axial, [18].

Figura 4.3 - Mapa de características modificado com a

variação das VIGVs, [11]. $\quad 45$

Figura 4.4 - Mapa de características do compressor utilizando VIGVs [3]. 45

Figura 4.5 - Deslocamento da linha de operação com o uso das

válvulas de alívio, [11].

Figura 5.1 - Mapa de Característica: razão de pressão versus

vazão mássica.

Figura 5.2 - Mapa de características da razão de pressão 
normalizada em função da vazão mássica corrigida para linhas de rotação obtidas a partir da rotina PCOMP.

Figura 5.3 - Mapa de características da eficiência isentrópica normalizada em função da vazão mássica corrigida para linhas de rotação obtidas a partir da rotina PCOMP.

Figura 5.4 - Mapa de características KU-V84: razão de pressão versus vazão mássica corrigida .

Figura 5.5 - Mapa de características KU-V84: Linha de rotação obtida pelo método de ponderação (Razão de Pressão versus Vazão Mássica Corrigida)

Figura 5.6 - Mapa de características KU-V84: Linha de rotação obtida pelo método de ponderação (Eficiência isentrópica versus Vazão Mássica Corrigida)

Figura 5.7 - Esquema do código PCOMP implementado

na ferramenta computacional NGGT.

Figura 5.8 - Interpolação linear bidimensional para a determinação do parâmetros que compõem o ponto de operação desejado.

Figura 6.1 - Mapa de Característica FRAME7: razão de pressão versus vazão mássica

Figura 6.2 - Mapa de Característica FRAME7: eficiência isentrópica versus vazão mássica

Figura 6.3 - Mapa de Característica GE7A: razão de pressão versus vazão mássica corrigida.

Figura 6.4 - Mapa de Característica GE7A: eficiência isentrópica versus vazão mássica corrigida.

Tabela 6.5 - Desvios do método de ponderação do PCOMP para a determinação da linha de rotação corrigida e normalizada 1,0 dos mapas de características da GE7A.

Tabela 6.6 - Desvios do método de ponderação do PCOMP para a determinação da linha de rotação corrigida e normalizada 1,019 dos mapas de características da GE7A.

Figura 6.5 - Mapa de Característica do compressor utilizado nas simulações realizadas por [13].

Figura 6.6 - Linha de rotação formada pela vazão mássica obtida utilizando os métodos Beta, Elipse e o método de ponderação do PCOMP Figura 6.7 - Mapa de Característica KU-84: eficiência isentrópica versus vazão mássica 
Figura 6.8 - Valores de eficiência isentrópica obtidos na saída do modelo PCOMP e aqueles referentes aos dados de operação da usina, apresentados na Tabela 6.8.

Figura 6.9 - Valores de vazão mássica obtidos na saída do modelo PCOMP e aqueles referentes aos dados de operação da usina, apresentados na Tabela 6.8. 


\section{Lista de tabelas}

Tabela 3.1 - Variação dos principais parâmetros termodinâmicos no estágio do compressor axial, [18].

Tabela 5.1 - Pontos referentes ao parâmetros de Razão de Pressão das linhas de rotação constantes conhecidas no mapa $\left(R P_{m}\right.$ e $\left.R P_{M}\right)$ e da linha ponderada.

Tabela 5.2 - Dados tabulados do mapa de características normalizado do compressor da turbina Siemens KU-V84.

Tabela 5.3 - Parâmetros da linha de rotação ponderada do mapa de características KU-V84 normalizado.

Tabela 6.1 - Desvios do método de ponderação do PCOMP para a determinação da linha de rotação corrigida e normalizada 0,95 dos mapas de características da FRAME7.

Tabela 6.2 - Desvios do método de ponderação do PCOMP para a determinação da linha de rotação corrigida e normalizada 1,0 dos mapas de características da FRAME7.

Tabela 6.3 - Desvios do método de ponderação do PCOMP para a determinação da linha de rotação corrigida e normalizada 1,05 dos mapas de características da FRAME7.

Tabela 6.4 - Desvios do método de ponderação do PCOMP para a determinação da linha de rotação corrigida e normalizada 0,985 dos mapas de características da GE7A.

Tabela 6.5 - Desvios do método de ponderação do PCOMP para a determinação da linha de rotação corrigida e normalizada 1,0 dos mapas de características da GE7A.

Tabela 6.6 - Desvios do método de ponderação do PCOMP para a determinação da linha de rotação corrigida e normalizada 1,019 dos mapas de características da GE7A.

Tabela 6.7 - Vazão Mássica obtida utilizando os métodos Beta, Elipse e o método de ponderação do PCOMP.

Tabela 6.8 - Dados de entrada do código desenvolvido provenientes dos dados de operação da planta, para cada ângulo de IGV.

Tabela 6.10 - Saídas do modelo PCOMP e desvios em relação aos dados da UTE 


\section{Nomenclatura}

$a$ Vazão mássica corrigida quando a razão de pressão é unitária (equação de elipse) / Fator de correção da vazão mássica

$b$ Razão de pressão para vazão mássica nula (equação de elipse) / Fator de correção da razão de pressão

c Constante arbitrária da equação de elipse / Fator de correção da eficiência

C Velocidade absoluta do fluido

$C_{a} \quad$ Velocidade axial

$C_{w} \quad$ Velocidade tangencial

$c_{P} \quad$ Calor específico a pressão constante

$h \quad$ Entalpia

IGV Inlet Guide Vane

$\dot{m} \quad$ Vazão mássica

$\frac{\dot{m} \sqrt{\theta_{1}}}{\delta_{1}}$ Vazão mássica corrigida semi-dimensional

M Número de Mach

$N \quad$ Velocidade de rotação do eixo

$\frac{N}{\sqrt{T}} \quad$ Velocidade de rotação corrigida semi-dimensional

p Pressão

$\frac{p_{02}}{p_{01}} \quad$ Razão de pressão adimensional

$\dot{Q} \quad$ Taxa de transferência de calor

$\dot{Q}_{E} \quad$ Vazão mássica na entrada do compressor

ROTENT Rotação corrigida de entrada da rotina de manipulação de mapas desenvolvida.

$R P \quad$ Razão de Pressão

RPENT Razão de Pressão de entrada da rotina de manipulação de mapas desenvolvida.

$T \quad$ Temperatura

$U \quad$ Velocidade da palheta

$V \quad$ Velocidade relativa do fluido 


$\begin{array}{ll}\dot{W} & \text { Taxa de transferência de trabalho } \\ w & \text { Trabalho específico } \\ z & \text { Curvatura da linha (equação de elipse) } \\ Z & \text { Parâmetro Beta de MacMillan }\end{array}$

Letras gregas

$\alpha_{1} \quad$ Ângulo de entrada do rotor

$\alpha_{2} \quad$ Ângulo de saída do rotor

$\beta_{1} \quad$ Ângulo de entrada do estator

$\beta_{2} \quad$ Ângulo de saída do estator

$\beta \quad$ Linha Beta

$\chi \quad$ Parâmetro genérico da linha de rotação

$\lambda \quad$ Fator de ponderação

$\eta \quad$ Eficiência

$\eta_{\text {iso }} \quad$ Eficiência isentrópica corrigida

$\theta_{1} \quad$ Parâmetros corrigido de temperatura

$\delta_{1} \quad$ Parâmetros corrigido de pressão

Índices

c Compressor

$i \quad$ Indica o ponto da linha de rotação em questão

$k$ Razão entre o calor específico a pressão constante e 0 calor específico a volume constante

$\begin{array}{ll}M & \text { Maior rotação } \\ m & \text { Menor rotação } \\ n & \text { dados do mapa original } \\ O D & \text { Off-Design } \\ s & \text { isentrópico } \\ 0 & \text { Propriedade de estagnação } \\ 1 & \text { Entrada do rotor } \\ 2 & \text { Saída do rotor } \\ 3 & \text { Saída do estator }\end{array}$

